\title{
Clinical Tips of Cesarean Section in Case of Breech, Transverse Presentation, and Incarcerated Uterus
}

\author{
Jun Takeda, MD, $\mathrm{PhD}^{1} \quad$ Gen Ishikawa, MD, $\mathrm{PhD}^{2} \quad$ Satoru Takeda, MD, $\mathrm{PhD}^{1}$ \\ ${ }^{1}$ Department of Obstetrics and Gynecology, Faculty of Medicine, \\ Juntendo University, Tokyo, Japan \\ 2 Department of Obstetrics and Gynecology, Chiba Hokusoh Hospital, \\ Nippon Medical School, Chiba, Japan \\ Address for correspondence Jun Takeda, MD, PhD, Department of \\ Obstetrics and Gynecology, Faculty of Medicine, Juntendo University, \\ 2-1-1, Hongo, Bunkyo-ku, Tokyo, Japan 113-8421 \\ (e-mail: jtakeda@juntendo.ac.jp).
}

Surg J 2020;6(suppl S2):S81-S91.

\begin{abstract}
Keywords

- breech presentation

- cervical elongation

- cesarean section

- incarcerated uterus

- transverse presentation

Cesarean section in breech or transverse presentation involves more complicated procedures than cesarean section in cephalic presentation because the former requires additional manipulations for guiding the presenting part of the fetus, liberation of the arms, and the after-coming head delivery; therefore, those cesarean sections are likely to be more invasive. Making a rather wide uterine incision to prevent uterine injury during delivery of the fetus facilitates smooth delivery of the fetus. Furthermore, in cases of breech or transverse presentation, it is important to initially identify the presenting part of the fetus and guide it to the incision opening in the lower uterine segment, because delivering the presenting part of the fetus first is a basic rule of delivery of the fetus. Smooth delivery of the fetus by means of breech extraction can prevent excessive stress or injury to the fetus. Therefore, it is important to acquire the knowledge and skills necessary to perform these techniques, including the internal version. Smooth delivery of the fetus is also less invasive for the mother because an extension of the uterine excision or injury to arteries and veins in the uterus and parametrium can be avoided. Incarcerated uterus occurring in cases of pregnancy with intrapelvic adhesion, endometriosis, cervical myoma, or extended cervix may result in excessive uterine and cervical injury when a transverse incision of the lower uterine segment is performed without caution. These conditions may result in difficulty in fetal delivery. Therefore, it is important to identify risks in advance and to choose the incision line with great care. Countermeasures for difficult delivery of the fetus need to be mastered by all practitioners of obstetrics. If the transverse incision fails to reach the uterine cavity, an inverted T-shaped or J-shaped incision should be made. Risks of complications such as injury to the cervical canal, the vagina, the bladder or ureter, and massive hemorrhage must be kept in mind.
\end{abstract}

\section{Cesarean Section in Case of Breech or Transverse Presentation}

\section{Preoperative Evaluation}

The presentation, position, and attitude of the fetus should be confirmed by transabdominal ultrasonography on admission and at departure from the ward for cesarean section. It is important to have images of the course of the surgery and delivery of the fetus by confirming the fetal position, location of the placenta and umbilical cord, and volume of amniotic fluid. The operation should be performed by imaging the descent, presentation, position, and attitude of the fetus through external examination according to Leopold's maneuver before laparotomy. Such imaging facilitates learning of the cesarean section techniques and obstetric management in the long run.
DOI https://doi.org/ 10.1055/s-0040-1702985. ISSN 2378-5128.
Copyright @ 2020 by Thieme Medical Publishers, Inc., 333 Seventh Avenue, New York, NY 10001, USA. Tel: +1(212) 760-0888.
License terms

()(1) $\Theta \circledast$ 
We basically use a transverse incision or a subumbilical longitudinal midline incision technique for skin incision. However, in cases of transverse or oblique presentation, a longitudinal incision is recommended by considering the possible extension of the incision wound. When performing a transverse incision, the Pfannenstiel incision is used as a rule, and the site of incision should be 3 to $5 \mathrm{~cm}$ above the pubic bone. It is important to secure the field of view; an incision at a higher site may cause difficulty in delivery of the fetus or may exacerbate uterine injury. If a higher incision site is required, caution should be exercised to secure the field of view for an incision of the rectus abdominis muscle, according to the original Maylard incision. In cases with a history of cesarean section or laparotomy or those with obesity, a sufficient field of view may be difficult to obtain; the selection and length of the skin incision line can affect the subsequent course of the operation.

\section{Tips and Warnings}

\section{Characteristics of Emergency Cesarean Sections in}

\section{Breech Delivery}

Emergency cesarean sections that have been switched from vaginal breech delivery involve the following specific characteristics: in most cases, the indication is nonreassuring fetal status caused by a sudden and rapid onset of cord compression due to a forelying or prolapsed cord; the baby must be delivered as quickly as possible; and it may be associated with rupture of the membranes and/or thin myometrium caused by effacement and elongation of the uterine isthmus because of progression in the descent of the presenting part (-Table 1). ${ }^{1}$ Thus, caution should be exercised during an incision of the myometrium because the fetus can be injured. To deliver the fetus, the operator grasps the fetal buttocks, hooking his/her index finger on the fetal bilateral groins, and pulls the fetal body gently, similar to the usual breech extraction maneuver. The femoral region must never be pulled with a finger over it. This can result in fetal femur fractures, which have been reported after cesarean breech delivery.

Obstetricians should understand and learn such characteristics of and strategies for cesarean delivery for breech presentation and establish a system to conduct an emergency cesarean section at any given time. For this purpose, obstetricians should consult with each other and form a multidisciplinary team that includes anesthesiologists, pediatricians, midwives, nurses, and paramedical staff to share information, cooperate immediately and closely, and receive simulation training. ${ }^{1,2}$

\section{Tips and Warnings}

Management of a Case with Cord Prolapse or NonReassuring Fetal Heart Rate Patterns

In cases of sudden onset of persistent fetal bradycardia and/or cord prolapse, vaginal examination should be performed to identify the causes and position of the cord. To relieve cord compression, the fetal presenting part should be elevated and tocolysis with nitroglycerin or ritodrine hydrochloride should be considered before transfer to the operating room. In most cases, the fetal heart rate is restored. Elevation of the presenting part using fingers through the vagina should be continued until delivery of the fetus. ${ }^{1}$

Table 1 Characteristics and risks of emergency cesarean sections for breech presentation

\begin{tabular}{|c|l|}
\hline 1 & In most cases, a poor fetal condition is an indication for an emergency cesarean section \\
\hline 2 & $\begin{array}{l}\text { A non-reassuring fetal status, which is common, is caused by a sudden and rapid interruption of the blood supply through the cord } \\
\text { due to a forelying or prolapsed cord }\end{array}$ \\
\hline 3 & $\begin{array}{l}\text { The presenting part descends disproportionately to the degree of cervical dilatation } \\
\text { - Delivery of the fetus is difficult and requires pushing of the presenting part upward through the vagina by an assistant } \\
\text { - A forcible procedure may tear the incision, resulting in increased bleeding } \\
\text { - Obstetricians must familiarize themselves with the procedures for cesarean breech delivery } \\
\text { - If the fetal head is entrapped, nitroglycerin is administered and/or the incision is extended to a J-shape or an inverted T-shape } \\
\text { if the fetus cannot be delivered because of a transverse-lying or other positions, the foot is grasped and the internal fetal version } \\
\text { is performed }\end{array}$ \\
\hline 4 & $\begin{array}{l}\text { The uterine isthmus elongates. } \\
\text { The incision should not be expanded upward in the direction where the fetal back or head is located } \\
\text { - An incision can likely be made at a lower location, such as the cervix or the vagina } \\
\text { - The fetus can be injured during the incision because of the thin myometrium }\end{array}$ \\
\hline 5 & Fetomaternal complications occur more frequently during emergency cesarean sections than during planned cesarean sections \\
\hline 6 & \begin{tabular}{l} 
A system to perform an extremely emergent cesarean section is needed \\
\hline
\end{tabular} \\
\hline
\end{tabular}




\section{Explanation of Procedures}

\section{Laparotomy}

Before the surgical procedure is initiated, the surgeon studies the images of the course of the operation and delivery of the fetus based on the findings obtained by ultrasonography in the ward or by external examination prior to the incision. The gravid uterus is not bilaterally symmetrical. In particular, the midline is difficult to find when left uterine displacement has been provided for preventing supine hypotensive syndrome or

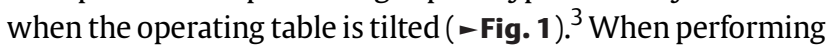
fasciotomy and peritoneotomy, caution should be exercised to avoid tearing of the abundant subfascial vessels. Before incision of the uterus wall, the position of presentation, leg position, and location and descent of the presenting part of the fetus should be confirmed by palpation from the serous surface of the uterus. Then, the uterine rotation status should be confirmed, and the incision line decided. This is useful for avoiding injury to the fetus and allows us to quickly reach the uterine cavity.

\section{Tips and Warnings}

Finally, the incision sizes of the skin and fascia should be confirmed at this point before a transverse incision is made on the lower uterine segment. An incision that is too small and excessive tension of the fascia and rectus abdominis muscle may cause difficulty during the delivery of the fetus. Therefore, the incision should be extended at this point, if necessary. After making a transverse incision of the lower

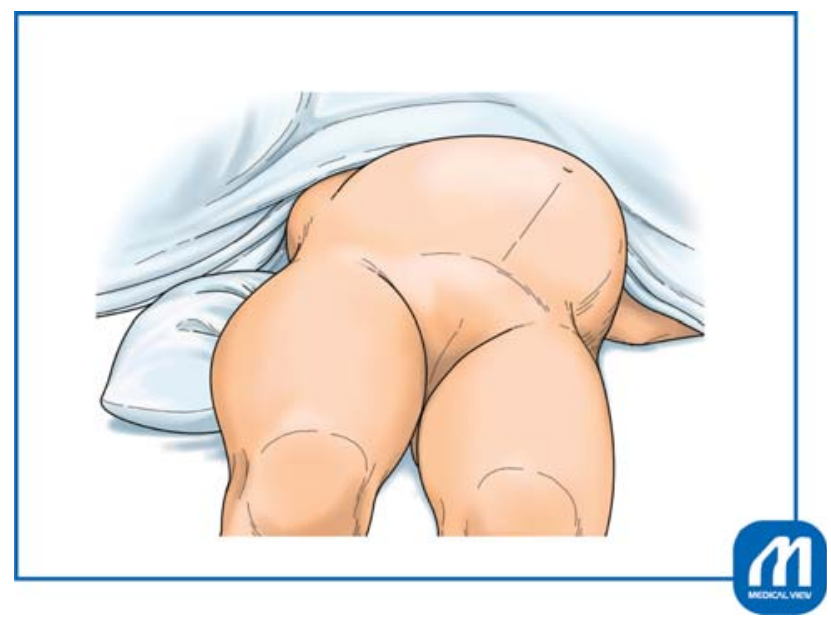

Fig. 1 Selection of the skin incision line. In cases of cesarean section, it is common to perform left uterine displacement by placing a pillow under the right side of the waist or by tilting the operating table immediately after inducing spinal subarachnoid anesthesia, aiming at preventing the occurrence of supine hypotensive syndrome. Because the surgical field is subsequently disinfected and the patient is draped, the midline becomes more difficult to identify. In addition, the abdominal skin is not bilaterally equal or symmetrical, depending on the position of presentation, fetal attitude, and rotation of the uterus. Therefore, the location of the skin incision line should be decided promptly but with care. (Reproduced with permission from Ishikawa G. Cesarean section for breech, transverse presentation and incarcerated uterus. In: Hiramatsu Y, Konishi I, Sakuragi N, Takeda S, eds. Mastering the Essential Surgical Procedures OGS NOW, No.3. Cesarean section. (Japanese). Tokyo: Medical View; 2010: 72-81. Copyright @ Medical View).

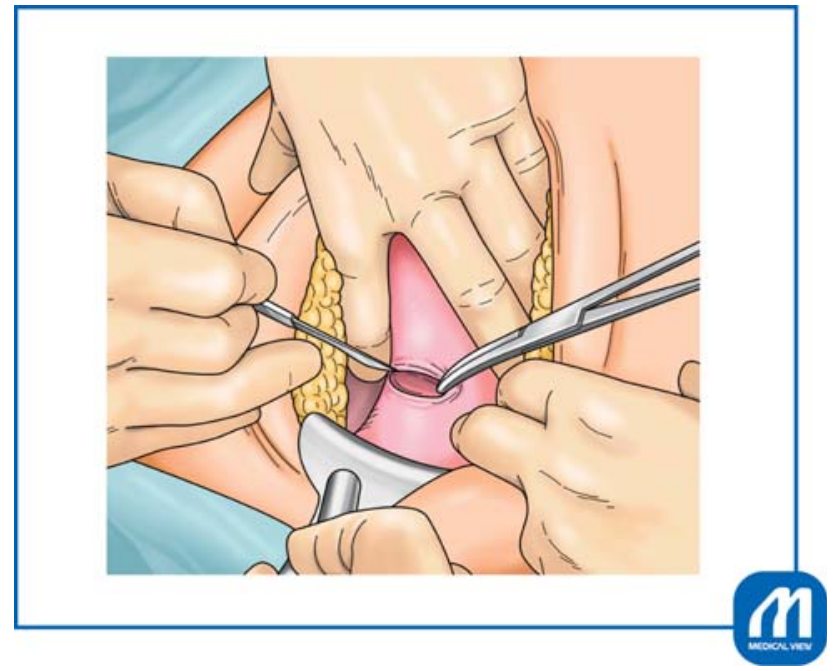

Fig. 2 Transverse incision in the lower uterine segment. While holding the lower uterine segment with two fingers of the left hand, the operator incises the lower segment with a round-edged knife to prevent bleeding and identify the location. The assistant aids in securing the field of view with the left hand and supports the wound surface with Pean forceps to facilitate a prompt incision. (Reproduced with permission from Ishikawa G. Cesarean section for breech, transverse presentation and incarcerated uterus. In: Hiramatsu Y, Konishi I, Sakuragi N, Takeda S, eds. Mastering the Essential Surgical Procedures OGS NOW, No.3. Cesarean section. (Japanese). Tokyo: Medical View; 2010: 72-81. Copyright @ Medical View).

uterine segment, the operation usually proceeds expeditiously enough that no modifications can be made.

\section{Incision of the Lower Uterine Segment}

To facilitate delivery of the fetus, the presenting part of the fetus should be touched from the serosal side of the uterus and manually elevated toward the maternal head. Then, the loose vesicouterine serosa is grasped with tweezers and incised transversely with scissors. The bladder is gently dissected from the underlying lower uterine segment. After this bladder separation, a transverse incision should be made in the lower uterine segment, in the same manner as in a cesarean section for a cephalic presentation (-Fig. 2) ${ }^{3}$ The incision made with a surgical knife to reach the uterine cavity can be extended manually or sharply with Cooper scissors (-Fig. 3 ). ${ }^{3}$ In either case, it is necessary to take care to avoid injury to the uterine artery and vein that lie on the extension line of the incision. Surgical techniques performed without due caution may cause additional injuries while guiding the fetus, liberating the arms, or after-coming head delivery in breech presentation, leading to major bleeding.

\section{Tips and Warnings}

A cesarean section in breech presentation involves more complicated procedures than a cesarean section in cephalic presentation because the former requires additional manipulations for guiding the presenting part of the fetus, liberation of the arms, and the after-coming 


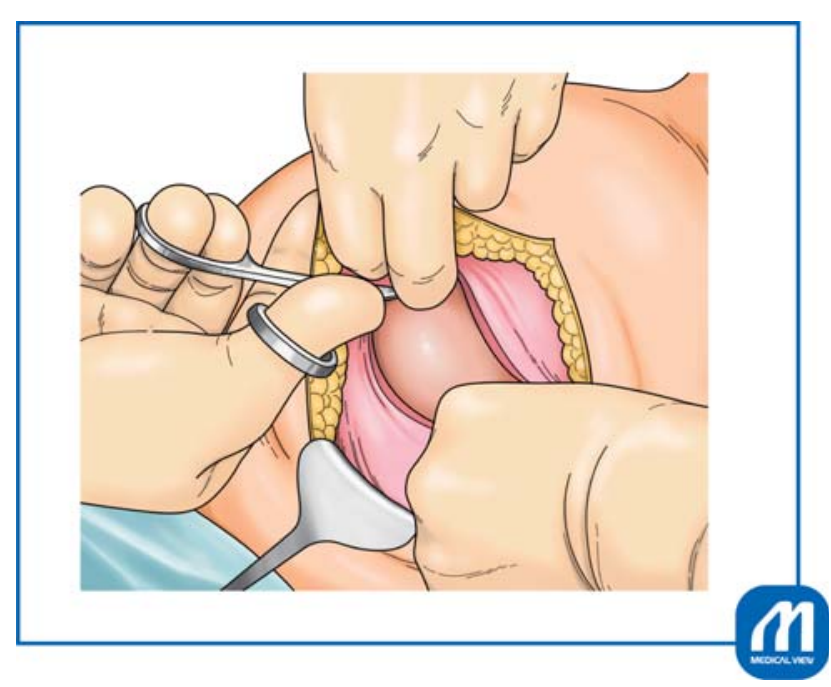

Fig. 3 Extension of the transverse incision in the lower uterine segment. In our institution, it is common to extend the uterine incision manually by "smiling up" the lateral apices. In cases of malpresentation, such as breech presentation at the cesarean section, Cooper scissors may be used, considering the possibility that subsequent delivery of the fetus might extend or injure the incision. The incision in the uterus is extended with Cooper scissors. Guiding with two fingers of the left hand, the lateral apices of the incision are cut in an arc shape toward the uterine fundus to make the "smiling up" incision. (Reproduced with permission from Ishikawa G. Cesarean section for breech, transverse presentation and incarcerated uterus. In: Hiramatsu Y, Konishi I, Sakuragi N, Takeda S, eds. Mastering the Essential Surgical Procedures OGS NOW, No.3. Cesarean section. (Japanese). Tokyo: Medical View; 2010: 72-81. Copyright ( $)$ Medical View).

head delivery. Therefore, a cesarean section in breech presentation is likely to be more invasive. Making a rather wide uterine incision to prevent uterine injury during the delivery of a fetus facilitates smooth delivery of the fetus. At this point, it is important to "smile up" the lateral apices of the incision in an arc shape toward the uterine fundus.

A "smile up" rather than a straight-line, incision is commonly used.

In a transverse or oblique presentation, smooth delivery of the fetus may be achieved by transverse incision of the uterine body based on the fetal presentation, as carefully determined in advance. The operator preoccupied with the lower uterine segment may make an incision in a narrow lower uterine segment, thereby possibly inducing injury at the incision site while guiding the fetus manually. Incising the lower segment is advantageous, considering the concerns of repeated cesarean section in the future and protection of the wound by suture and repair after incision of the peritoneal reflection of the vesicouterine pouch. However, a transverse incision of the uterine body might be more advantageous with regard to the patency of the guiding path for the fetus. A J-shaped or U-shaped incision may be superior on the assumption that the fetus in oblique or transverse presentation will be delivered without rotation (-Fig. 4) ${ }^{1,4}$ However, if an incision is made at a high position without considering the positional relationship between the fetus and uterus, it may be difficult to guide the fetal buttocks located at a lower position to the incised opening.

\section{Delivery of the Fetus}

The presenting part of the fetus should be identified by finding the fetus from the incision in the uterus. In cases of footling presentation, the operator should hold the legs
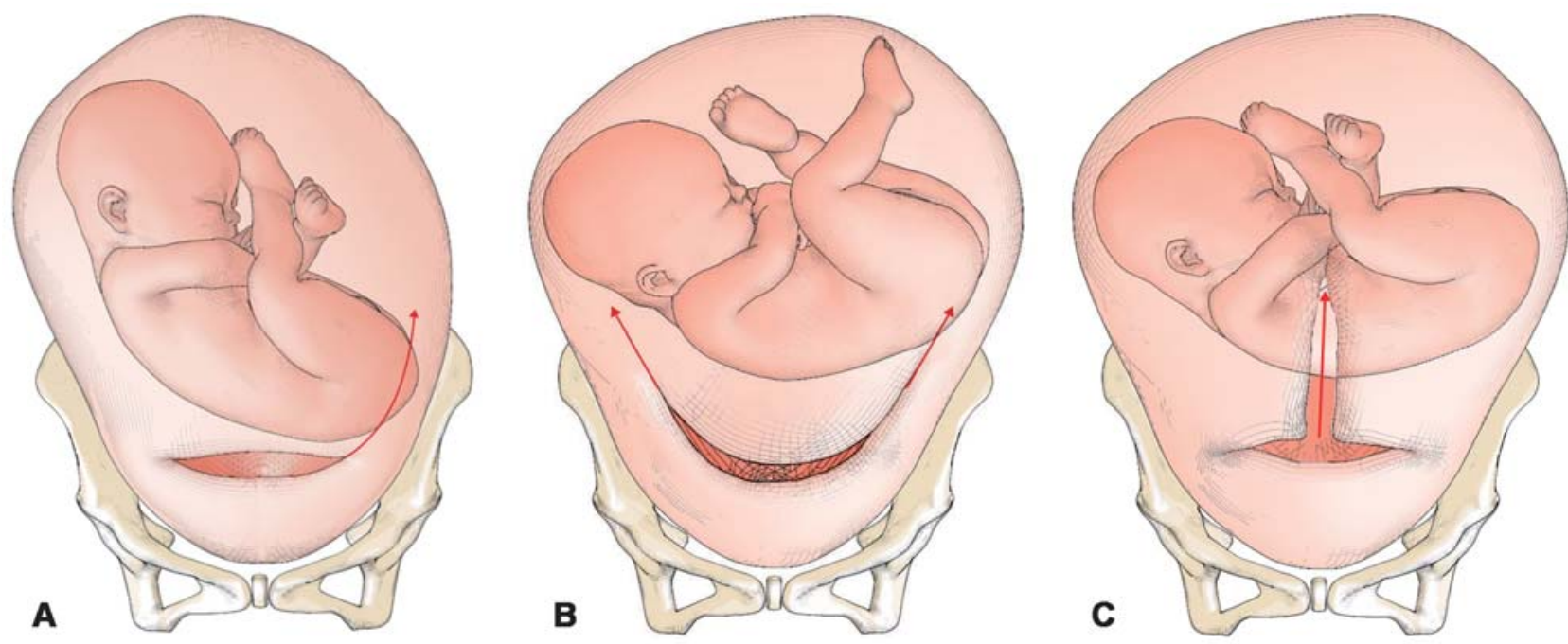

Fig. 4 Extension of the uterine incision. A slightly wider incision is made into the myometrium. For a difficult delivery, the incision is preferably extended upward to a J-shape (A) or U-shape (B) or an inverted T-shape (C). If a transverse incision does not reach the uterine cavity such as uterine incarceration, the incision should be extended upward to make a s shape or be extended into an inverted T shape. (Reproduced with permission from Takeda S. Important point of emergency cesarean section. In: Takeda S, Makino S, Takeda J, eds. Management of Breech Delivery and Shoulder Dystocia. (Japanese). Tokyo: Medical View;2019:106-110. Copyright @ Takeda S). 
and proceed to the subsequent guiding process. Because holding the legs and subsequent guiding and extraction place a burden on the joints of the lower limbs (ankle, knee, and hip joints) of the fetus, it is recommended that both hips be held if possible. In cases of complete or frank breech presentation, both hips (iliac crest to inguinal region) should be held. In cases of fetal presentation in which the fetal back is facing the maternal back (sacrum posterior positions: sacrum posterior, left sacrum posterior, and right sacrum posterior), the fetal back should be guided to rotate around the long axis of the fetal body toward the maternal ventral side, to allow the spinal column in the fetal back to move toward the anterior portion of the maternal body (sacrum anterior position). This corresponds to the position of presentation at the end of the second rotation during vaginal delivery in breech presentation. This maneuver is advantageous in that it facilitates liberation of the arms and delivery of the fetal head following delivery of the fetal legs and trunk. Furthermore, this maneuver allows the passage of the fetus to more appropriately correspond to the incisional opening of the uterus.

\section{Tips and Warnings}

For identifying the lowest presenting part of the fetus, four fingers (index to little fingers) of one hand (on the maternal caudal side) of the operator, in principle, are initially used. Later, the fetus should be held mainly with two or three fingers, that is, the index finger, (middle finger) and thumb, of both hands. There is concern that careless holding with five fingers may result in extension of the uterine incision and injury to the uterine artery and vein. When the operator is not skilled enough in performing cesarean section in breech presentation or when a sufficient abdominal incision or uterine incision is lacking (such as in patients with a history of surgery or obesity), the operator is apt to apply excessive force when attempting to hold the fetus. It is useful to place gauze between the holding fingers and fetus to prevent slippage. This allows the fetus to be held more firmly and facilitates smooth delivery of the fetus. If the presenting part descends deeply, the assistant manually elevates the presenting part through the vagina and then the operator attempts to deliver the infant.

In case of transverse or oblique presentation, since the presenting part is not often palpable, the operator probes the foot to grasp and deliver the infant. To touch the foot, the operator rotates the fetus, using fingers inserted through the vagina and placed on the external uterine wall. If the foot is palpable, the operator should grasp the ankle (-Fig. 5). ${ }^{1}$ Grasping of the lower legs or thighs may cause fractures. If the foot is difficult to find, extending the incision of the myometrium in the direction where the foot may be located would facilitate grasping of the foot for performing the internal version. If the delivery is difficult, one should not

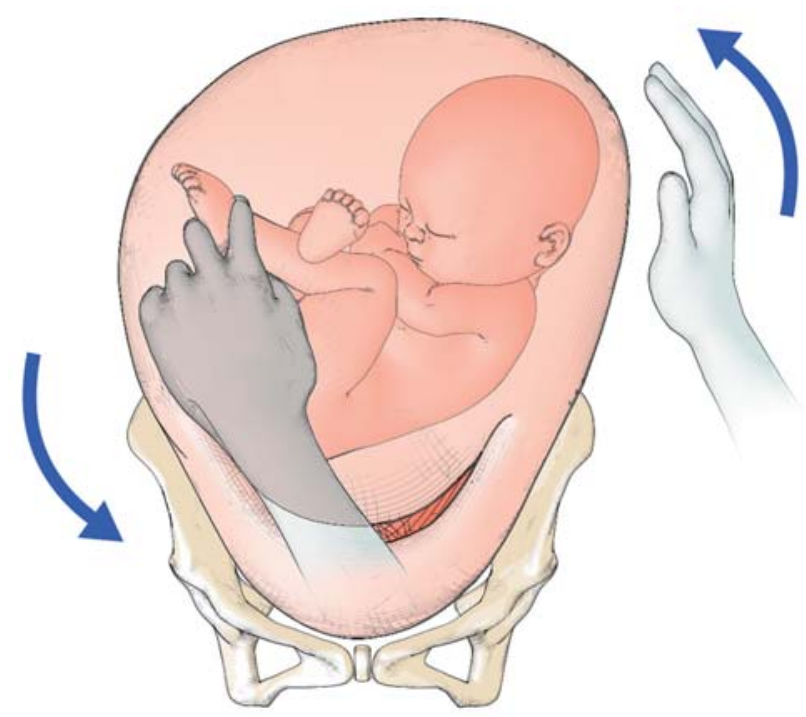

Fig. 5 Internal version technique by grasping of the fetal feet. The operator grasps the fetal ankle over the medial and lateral malleoli with three fingers (thumb, index, and middle fingers), pulling toward the incision. Simultaneously, the operator leads the fetal buttocks to the incision, placing the hand on the external uterine wall. ${ }^{1}$ (Reproduced with permission from Takeda S. Important point of emergency cesarean section. In: Takeda S, Makino S, Takeda J, eds. Management of Breech Delivery and Shoulder Dystocia. (Japanese). Tokyo: Medical View;2019:106-110. Copyright @ Takeda S).

hesitate to extend the incision to a J- or U-shape, or an inverted T-shape to facilitate the delivery (-Fig. 4). ${ }^{1,4}$ There is a question as to whether the fetal head or the buttocks should be guided as the presenting part. Because the hips and legs of the fetus are easier to hold and guide than the fetal head, the buttocks are guided as the presenting part in principle. However, there is no concern as to setting the fetal head as the presenting part if smooth manipulation is secured. In this case, delivery of the fetus is in accordance with a cesarean section in cephalic presentation.

\section{Tips and Warnings}

In cases of transverse or oblique presentation, the operator's hand tends to be inserted deeply into the uterus. Therefore, more attention is needed to avoid extension of the uterine incision or injury to the uterine artery and vein in transverse or oblique presentation than in breech presentation. Namely, careless manipulation during manual exploration and rotation of the fetus may result in extension of the uterine incision, injury to lateral blood vessels of the uterus, or laceration on the cervical side of the uterine incision. Due caution is necessary to avoid these problems.

The presenting part should be guided to the incision opening of the uterus and extracted toward the maternal feet while holding both fetal hips securely. The goal is to keep the fetus horizontal while extracting the fetus until the 


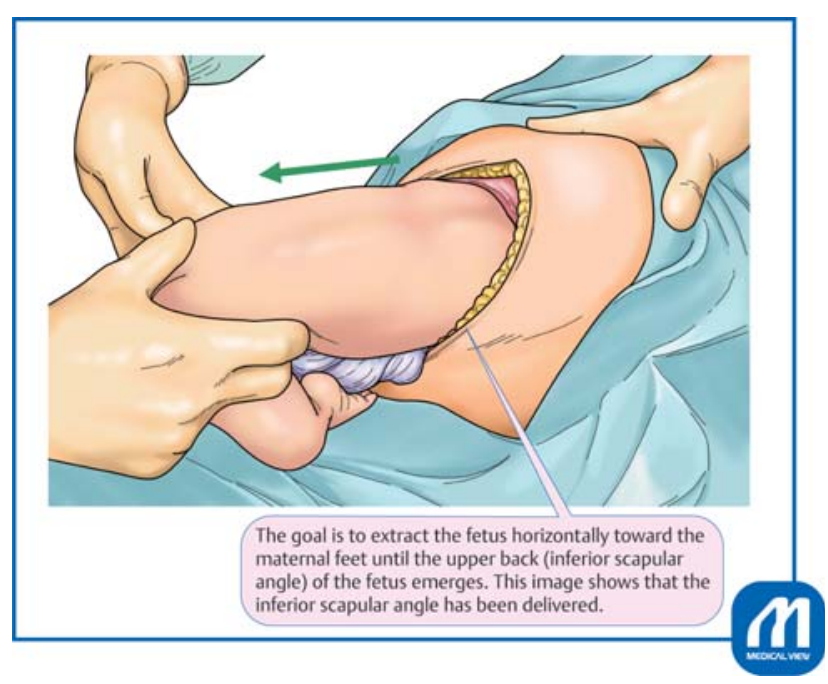

Fig. 6 Delivery of the fetus. The presenting part of the fetus is extracted while holding both fetal hips securely. If the fetus is deflected or elevated upward with excessive consideration of the subsequent delivery of the fetal head, it increases the risk of injuries at the incision, such as extension of the uterine incision. (Reproduced with permission from Ishikawa G. Cesarean section for breech, transverse presentation and incarcerated uterus. In: Hiramatsu Y, Konishi I, Sakuragi N, Takeda S, eds. Mastering the Essential Surgical Procedures OGS NOW, No.3. Cesarean section. (Japanese). Tokyo: Medical View; 2010: 72-81. Copyright $\odot$ Medical View). upper back (inferior scapular angle) of the fetus is delivered (-Fig. 6). ${ }^{3}$

In cases of footling presentation, the fetus is initially guided and extracted while holding both legs, and then the hip (iliac crest to inguinal region), of the fetus. However, in cases of breech presentation, the hip of the fetus is held basically from the beginning. In cases of complete breech presentation, the operator may be tempted to guide the fetal legs first, but caution is necessary because there is concern that the uterine incision might be damaged because of the process by which the fetal legs bent at the hip and knee joints extend during passage through the incisional opening. If the fetus is guided while holding the hips after elevating the lowest part of the fetus toward the maternal head, a frank breech presentation in the uterus may be obtained, leading to smooth delivery of the trunk.

After delivering the fetus horizontally toward the maternal feet until the inferior scapular angle emerges, the operator should proceed to deliver the fetus in the manner of the Bracht maneuver for vaginal delivery in breech presentation (-Figs. 6, 7). ${ }^{3}$ Namely, the fetal trunk should be elevated and dorsiflexed (-Fig. 7A), ${ }^{3}$ and then rotated toward the maternal ventral side in an arc with the uterine incision serving as the fulcrum (-Fig. 7B). ${ }^{3}$

When the arms are not brought down by the Bracht maneuver during delivery of the fetus, the procedures of liberation of the arms and delivery of the after-coming head

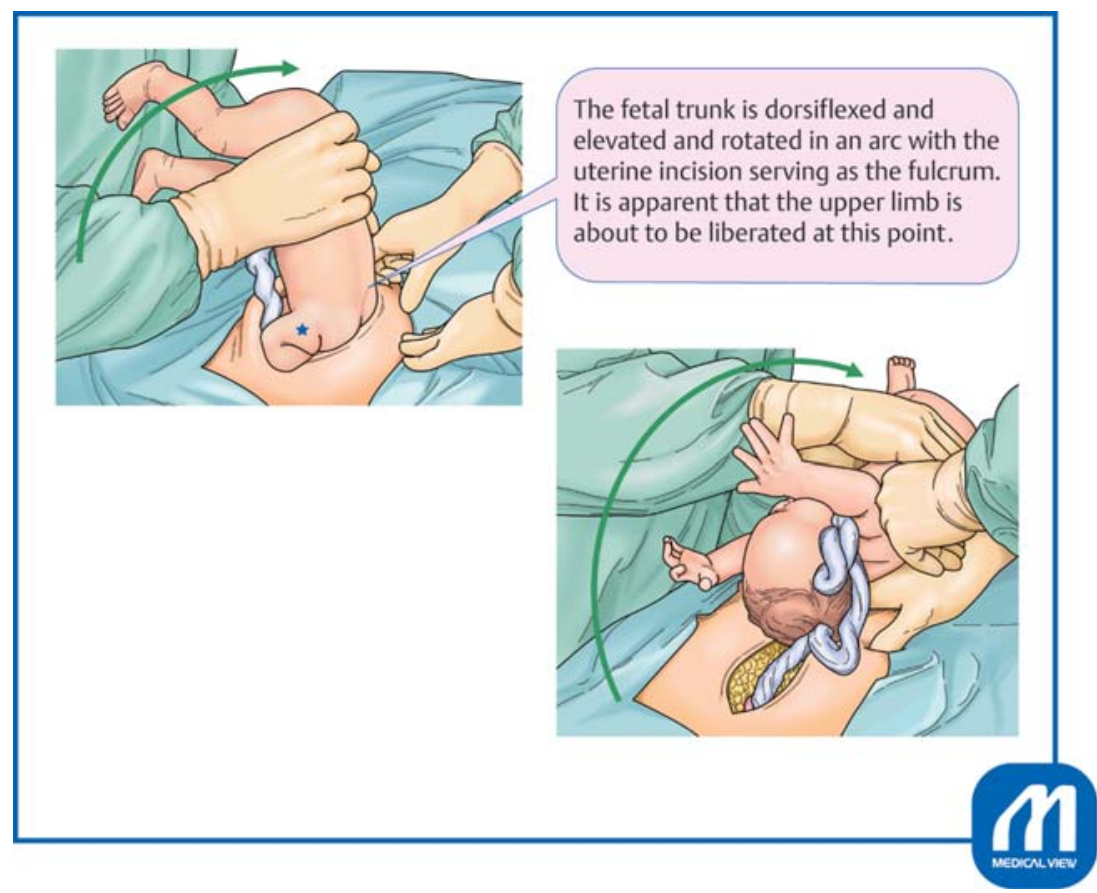

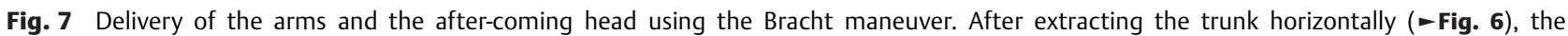
operator should proceed to liberation of the arms and delivery of the fetal head in the manner of the Bracht maneuver (A, B). The operator should try to achieve smooth delivery without interruption following delivery of the trunk. Whenever liberation of the arms is performed using the classic technique, with delivery of the fetal head according to the Mauriceau maneuver, these manipulations should be conducted smoothly at a constant pace and in a consistent manner with flexibility, as needed, to adapt to circumstances. (Reproduced with permission from Ishikawa G. Cesarean section for breech, transverse presentation and incarcerated uterus. In: Hiramatsu Y, Konishi I, Sakuragi N, Takeda S, eds. Mastering the Essential Surgical Procedures OGS NOW, No.3. Cesarean section. (Japanese). Tokyo: Medical View; 2010: 72-81. Copyright ( $)$ Medical View). 
should be performed. In cases of difficult delivery, the arms should be liberated in the manner of the classic method of liberating the arms. More specifically, while the fetal trunk is elevated anteriorly to the maternal body, the arm emerging on the maternal feet side of the uterine incision opening should be liberated with the index finger hooked in the fetal elbow joint. After both arms are liberated in this manner, the fetus should be kept dorsiflexed and elevated anteriorly to the maternal body; the after-coming head is subsequently delivered ( - Fig. 7B). ${ }^{3}$ In cases of vaginal delivery in breech presentation, the fetal back is rotated laterally to the maternal body, and the arms located posteriorly are liberated in the first and second breech presentations. In cases of cesarean section, uniaxial rotation of the fetus should not be performed by exerting force. This is because the uterine incision wound extends in the horizontal direction of the maternal body, and the mode of spread of the birth canal is different from that in vaginal delivery in breech presentation. The key to successful liberation of the arms is to guide the arm to be liberated in the manner of wiping the forehead of the fetus with the palmar side of the arm. If the arm is initially guided without due caution in the downward direction, the uterine incision may be damaged by the fetal elbow, the fetal upper arm may be injured as fracture, or an excessive burden may be imposed on joints of the fetus. Although successful liberation of the arms usually leads to delivery of the aftercoming head without difficulty, delivery of the fetal head is assisted by the Mauriceau or Veit-Smellie maneuver. The fetal trunk is already dorsiflexed and elevated anteriorly to the maternal body by the Bracht maneuver, showing the occipital protuberance of the fetal head. Therefore, it is not necessary to perform extraction posteroinferiorly to the maternal body according to the Mauriceau maneuver during vaginal delivery in breech presentation.

When the head is entrapped, the incision should be extended to a J-shape to deliver the infant. If time permits, administration of nitroglycerin can release the myometrial tone to enable a smooth delivery. In cases of cesarean delivery of preterm infants and those with a sufficient descent of the head or breech presentation, the anesthesiologists should be alerted beforehand to prepare nitroglycerin.

After removal of the placenta, the cervical os should be observed. If the cervical os is closed, it should be dilated during or after the operation to allow the lochia to flow outward. The uterine cavity should be bluntly removed using gauze to eliminate retained membranes.

\section{Tips and Warnings}

If liberation of the arms and delivery of the after-coming head are found to be difficult, the operator should not hesitate to attempt extension of the uterine incision. It is important to extend the incision in an upward direction toward the uterine fundus as a J-shaped incision to prevent injury to the uterine artery and vein. It may also be helpful to add an incision at the center of the uterine incision toward the uterine fundus to make an inverted Tshaped incision. It is recommended that the decision to perform extension of the uterine incision or add an inverted T-shaped incision should be made in advance as the second-best procedure, which should be performed without hesitation by the operator or the first assistant in the event of a difficult delivery. Continuation of surgical manipulations, without sufficient caution, which reduces the success in liberation of the arms and delivery of the fetal head, may unnecessarily exacerbate injury to the uterine incision and result in complications. The J-shaped and inverted T-shaped incisions are methods that allow for extension of the incision, but do not cause injury to the incision. They not only facilitate delivery in difficult cases but also prevent maternal injury, thereby leading to decreased surgical stress to the maternal body and decreased blood loss.

\section{Closure of the Abdomen}

The uterine incision wound should be closed by suture using 0-synthetic absorbable suture, such as Vicryl or Monocryl. The interrupted suture or Z-suture on the bilateral cut end should be performed for ligation of thick branches of the uterine artery and prevention of dead space or hematoma. The uterine incision is closed basically with two layers. The first layer is sutured by employing interrupted sutures such that both endometrial layers meet precisely. Continuous sutures on the inner side of the uterine wall are not employed because a history of continuous sutures on the inner side of the uterine wall might influence the development of placenta accreta in patients with prior cesarean section. ${ }^{5}$ The second layers may be sutured by employing a continuous interlocking suture or interrupted sutures.

There is no need for suturing the serosa on the vesicouterine pouch to prevent elevation and adhesion of the bladder, covering over the uterine wound. Douglas pouch, both sides of the peritoneal cavity, and the vesicouterine pouch should be examined to determine the hemostasis state and presence/absence of any abnormalities. The uterine adnexa should also be checked for any lesions or abnormalities.

The peritoneal cavity is sufficiently irrigated with 2000 to $3000 \mathrm{~mL}$ of physiological saline. A continuous closed suction drain is inserted into the Douglas pouch, if needed.

Absorbable adhesion barrier, such as Seprafilm, is applied to the wounds on the uterus and abdominal wall to prevent development of subsequent adhesion. The serosa, fascia, subcutaneous tissue, and epidermis are sutured to close the wound. The patency of the uterine os and outflow of lochia are confirmed by speculum and pelvic examinations. Then, the position of the uterine fundus and propriety of uterine contractions are confirmed to complete the operation. 


\section{Cesarean Section in Case of Incarcerated Uterus or the Cervical Elongation}

\section{Preoperative Preparation}

In cases of cesarean section for an incarcerated uterus, the success of the operation depends on whether cervical elongation and retroverted uterus can be detected preoperatively and whether the cesarean section procedure is smooth and minimally invasive to the maternal body. Incarceration of the uterus may not be recognized in early pregnancy. However, when extreme anterior-upward displacement of the cervix of the uterus or globular tumorous sensation in the posterior vaginal fornix is found by vaginal examination on admission for cesarean section or preoperative examination before departure from the hospital ward, the uterine cervix located anterosuperior to the bladder should be further examined by transabdominal ultrasonography to determine whether there is either elongation of the cervix or incarceration of the uterus. The height of the lower uterine segment and site of the uterine cavity should be examined by ultrasonography to decide the cutting level of the uterus before the operation.

If a diagnosis of the incarcerated uterus was made at term, elective cesarean section should be performed with special attention. When a cesarean section is performed without the recognition of an incarcerated uterus, it might be difficult to deliver the fetus because it will not reach the uterine cavity and consequently result in fetal asphyxia. Several complications such as complete cutting of the cervix or the vagina, extended uterine incision, incision on the posterior wall of the uterus through the vagina, or laceration due to massive hemorrhage may occur, because the lower uterine segment is dislocated extremely to the upper site compared with that in an ordinary cesarean section..$^{3,4,6}$ Therefore, a strategy for a safe cesarean section to avoid special complications of incarceration should be developed.

The incarcerated gravid uterus is recognized as being in a state of uterine retroflexion by pelvic examination or transvaginal ultrasonography in early pregnancy. As gestational weeks increase, the degree of retroflexion increases, resulting in embedding of the uterine fundus in the Douglas' pouch at the end of pregnancy (-Fig. 8). ${ }^{4}$ When there is elongation and elevation of the cervix due to cervical myoma in the posterior wall of the uterus or myoma in the lower uterine segment, cervical findings are similar to those in cases with an incarcerated gravid uterus (-Fig. 9). ${ }^{4}$

There are characteristic pelvic examination findings; the uterovaginal region is deviated anteriorly and superiorly to the pubis to an extreme degree. The protruded uterus and myoma are palpable in the Douglas' pouch. Colposcopy may fail to identify the uterovaginal region because it is deviated superiorly. It is common for the uterine fundus to be lower according to the gestational week, causing elongation of the bladder.

If the incarcerated gravid uterus is not recognized until the end of pregnancy, pelvic examination may raise suspicion because the uterovaginal region is deviated anteriorly and superiorly to an extreme degree, although the cervix is difficult to identify on transabdominal ultrasonography. ${ }^{7}$

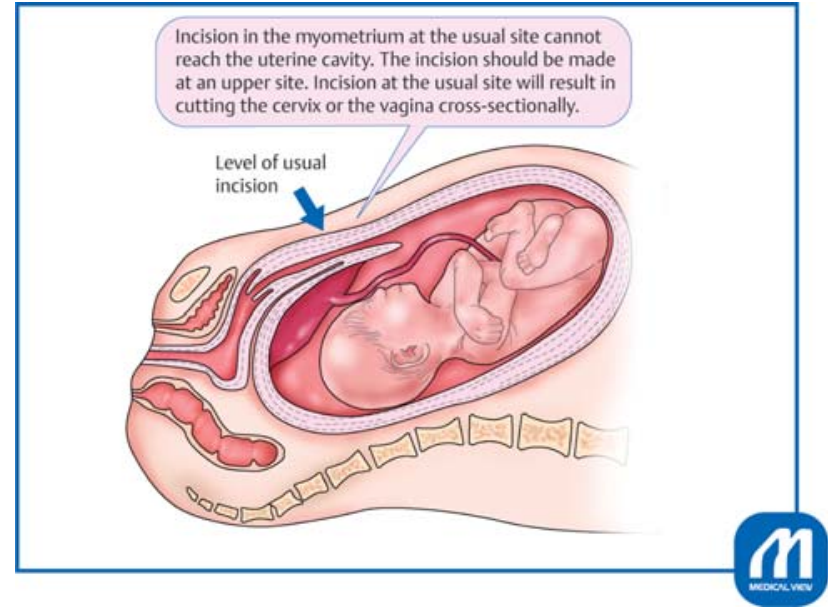

Fig. 8 Incarcerated uterus. Pelvic examination readily leads to a diagnosis of incarcerated gravid uterus because this examination yields characteristic findings. However, it is likely to be overlooked on ultrasonography alone. Because the cervix and uterine wall are thin at the end of pregnancy, it is difficult to diagnose retroflexion and incarceration of the uterus. The placenta attached to the posterior wall can be misdiagnosed as low-lying placenta or placenta previa. (Reproduced with permission from Takeda S. Cesarean section for incarcerated uterus and elongation of the uterine cervix. In: Hiramatsu Y, Konishi I, Sakuragi N, Takeda S, eds. Mastering the Essential Surgical Procedures OGS NOW, No.10. Massive Obstetric Hemorrhage: Critical Care for Intractable Bleeding and Definite Strategies of Hemostasis. (Japanese). Tokyo: Medical View; 2012: 154-159. Copyright (c) Medical View).

On ultrasonography, the cervix is deviated anteriorly and superiorly, and the bladder appears to be suspended upward. ${ }^{7}$ If incarcerated gravid uterus is suspected, magnetic resonance imaging can provide a definitive diagnosis based on the locations of the vagina, cervix, and bladder in the sagittal view. ${ }^{8}$ It is important to determine the level of the skin incision and the site of the uterine incision wound in advance, by confirming the level of entry into the uterine cavity by ultrasonography prior to implementation of cesarean section. $^{3,4}$ Intraoperative ultrasonography is also useful.

\section{Tips and Warnings}

Pathophysiology of the Incarcerated Uterus

Pregnancies complicated by myoma, endometriosis, and/or pelvic adhesion have been increasing with recent tendencies toward later marriages, increased pregnancy rates in women of advanced maternal age, and advancements in fertility treatments. Along with these trends, elongation of the uterine cervix has been attracting close attention as a reason for near-misses during cesarean section. Related near-miss cases, such as delivery of the fetus from the posterior wall of the uterus after incising the cervix and vaginal wall, failure to reach the uterine cavity during uterine myometrium incision, and massive hemorrhage, have been reported. Although uterine retroflexion before 12 gestational weeks is found at a frequency of 11 to $19 \%$ (15\%), incarcerated gravid uterus is 


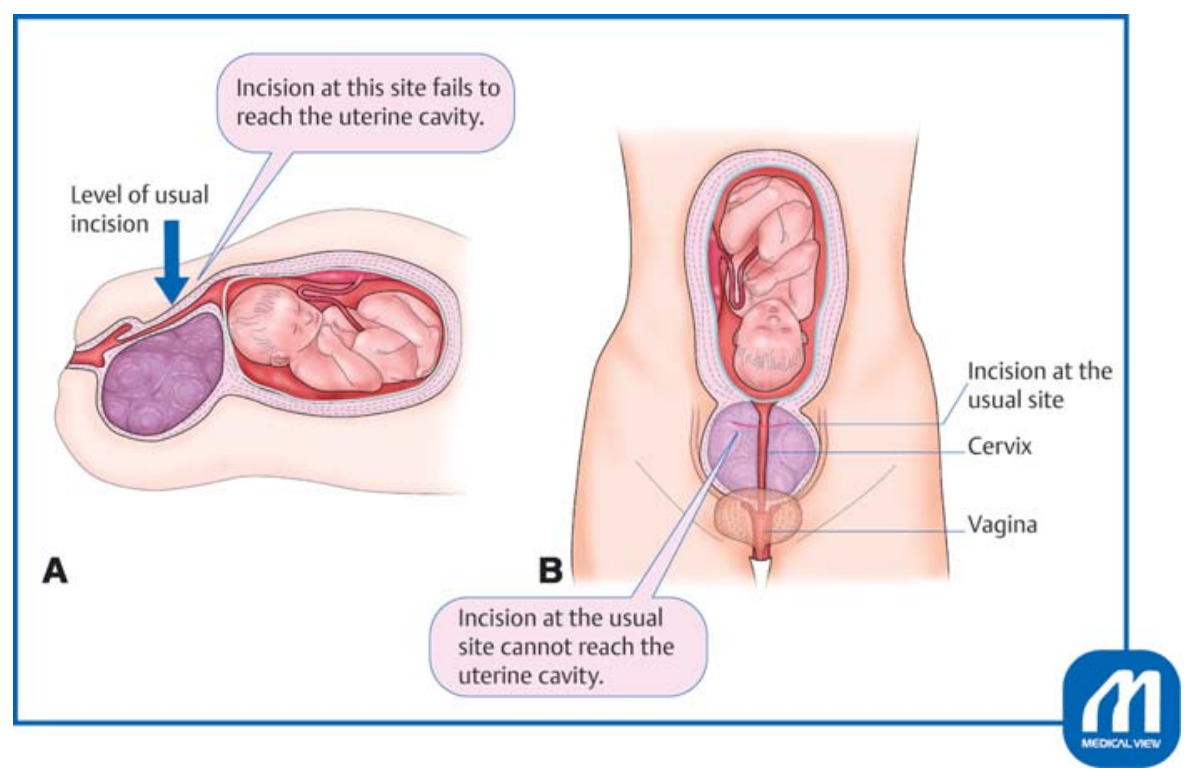

Fig. 9 Cervical elongation due to a myoma in the cervix. (A) In cases with cervical myoma in the posterior wall of the uterus or myoma in the lower uterine segment, the cervix is elongated. Therefore, myometrial incision at the usual level fails to reach the uterine cavity. In particular, in the case of performing a Pfannenstiel transverse incision, upward deviation of the lower uterine segment may be overlooked because the field of view in the peritoneal cavity is limited. Therefore, a transverse incision in the myometrium at the usual site fails to reach the amniotic cavity and the cervix is cut instead. (B) Incision at the usual site will result in cutting the cervix cross-sectionally or cutting into myoma in the posterior wall. (Reproduced with permission from Takeda S. Cesarean section for incarcerated uterus and elongation of the uterine cervix. In: Hiramatsu Y, Konishi I, Sakuragi N, Takeda S, eds. Mastering the Essential Surgical Procedures OGS NOW, No.10. Massive Obstetric Hemorrhage: Critical Care for Intractable Bleeding and Definite Strategies of Hemostasis. (Japanese). Tokyo: Medical View; 2012: 154-159. Copyright @ Medical View).

recognized in 1 out of 3,000 pregnant cases. ${ }^{7}$ Risk factors include pelvic inflammatory disease, adhesion after gynecological surgery, endometriosis, myoma in the posterior wall of the uterus, and congenital anomalies of the uterus. ${ }^{7}$

There are complications such as injury to the bladder or ureter, difficulty delivering the fetus, cervical incision, vaginal wall incision, and delivery of the fetus from the posterior wall of the uterus. Massive hemorrhage resulting in hysterectomy has also been reported.

Because uterine ruptures resulting from an attempt at vaginal delivery have occurred in the past, the current rule is that cesarean section should basically be selected for delivery in all cases with an incarcerated gravid uterus.

Symptoms become prominent after 13 to 17 gestational weeks, varying from none to pollakiuria, urinary retention, dysuria, incontinence, abdominal pain, suprapubic pain, low back pain, feeling of anal pressure, feeling of abdominal distension, and constipation. Symptoms may be intermittent or improve in some cases.

\section{Tips and Warnings}

\section{Pitfalls}

If the same doctor is in charge of ongoing medical check-ups of the patient from early in the pregnancy, it is relatively easy for the doctor to diagnose incarcerated gravid uterus, and cesarean section can be performed with this risk in mind. However, if multiple doctors see this patient, as in university hospitals, a problem may arise. Namely, uterine retroflexion might be missed as the uterus grows larger because of poor processes when one doctor takes over from another.

If transvaginal ultrasonography alone is used for medical check-ups of pregnant women, without performing the proper pelvic examinations, incarcerated gravid uterus may be overlooked. Placenta attached to the posterior wall of the uterus may be misdiagnosed as placenta previa or low-lying placenta.

In pregnancy with uterine myoma, cervical myoma and myoma in the lower uterine segment can cause the problem of cervical elongation. In particular, in cases with myoma in the posterior wall of the uterus, it is possible for cesarean section to be performed without due caution because there is no myoma at the site of incision in the myometrium. However, problems may arise during surgery because the cervix might be unexpectedly elongated.

It is common for the posterior wall of the uterus to be firmly adherent to the Douglas pouch in cases with an incarcerated gravid uterus, and the uterus may not be elevated outside the body cavity at the time of myometrial suture. If the wound area is elongated or the cervical canal is cut inadvertently, it is difficult to secure the field of view, and this results in difficulty with suturing. Suture during heavy bleeding and suture of the uterine wound without orientation may result in misalignment of the wound edges or failure in achieving clean closure of the wound. 


\section{Explanation of Procedures}

\section{Laparotomy}

Cesarean section for an incarcerated uterus is basically the same as a routine cesarean section. However, to facilitate subsequent surgical manipulations, a longer than usual incision should be made to provide a large field of view. A subumbilical longitudinal midline skin incision is preferred because of a wider field of view. The possibility that the incision is cut upward to the navel during the operation should be assumed.

When the cervix is elongated because of myoma, the positional relationships between the myoma, the cervix, the uterine body, and the round ligament of the uterus, and the bladder need to be confirmed. The bladder may be located extremely low but can also be elevated. The bladder should be palpated directly, and the urethral catheter be confirmed. If the bladder is extremely elevated, the upper end must be confirmed and separated.

\section{Uterine Incision}

As for incision in the myometrium, the peritoneal reflection of the vesicouterine pouch cannot be the target, as would routinely be the case, and the boundary between the uterine body and isthmus is ambiguous. Therefore, we can rely only on preoperative ultrasonographic findings in such cases.

The location of the uterine cavity should be confirmed by intraoperative ultrasonography, whenever possible. Because the vaginal wall and cervical canal in the final stages of pregnancy are thin, it may not be possible to confirm the location of the internal cervical os. It is also important to make sure of the distance to the uterine cavity. There is no need to strictly adhere to the use of deep transverse incision. If the cervix and the lower uterine segment are not distinguishable, longitudinal uterine incision is also a reasonable choice. Depending on the length of the elongated cervix, a longitudinal or transverse incision is made in the muscular layer near the umbilical region. When a transverse incision does not reach the amniotic cavity, the incision should be extended upward in the direction toward the uterine body in the manner of an inverted T-shaped or J-shaped incision.

\section{Tips and Warnings}

\section{In Cases of Failure to Reach the Uterine Cavity}

If pregnancy progresses with the uterus in an incarcerated state, the uterine cervix becomes extended and elongated. In this case, if a uterine incision is made in the peritoneal reflection of the vesicouterine pouch, it enters the cervical canal. As a result, delivery of the fetus cannot be achieved, and the paracervical tissue or posterior wall of the uterus may be incised erroneously, leading to massive hemorrhage. The cervical canal may also be cross-sectioned. In any event, the operator may lose orientation and become disconcerted.

If the uterine cavity is not reached by incising the expected thickness of the myometrium, an inverted T-shaped or J-shaped incision should be made from the midline without hesitation, keeping this condition in mind
(-Fig. 4). When cutting upward, the intestinal tract should be protected using a bladder spatula or intestinal spatula. If the uterine cavity is reached, the fetal membranes will bulge and may even rupture. Because the fetus is to be delivered from the lower part, rupture may occur unless a wide incision is made.

If the incision is deepened without recognizing this condition, the uterine cavity is reached from the posterior wall of the uterus. There is a report of the fetus being delivered twice via the vagina. ${ }^{6}$ There are also cases in which the cervical canal was cut cross-sectionally without realizing that this had taken place or in which the fetus was delivered from the posterior wall of the uterus after partial incision.

\section{Delivery of the Fetus}

In cases of cephalic presentation, cephalic delivery should be performed by employing a mild Kristeller maneuver via the incision opening of the uterus. In cases of breech presentation, the trunk should be delivered according to the cesarean section in breech presentation procedure, followed by liberation of the arms and delivery of the fetal head. These procedures are the same as those in cesarean section in breech or transverse presentation, but the patency of the uterine os is more difficult to confirm.

\section{Closure of the Abdomen}

The myometrium should be sutured by placing simple interrupted sutures with absorbable thread. The second layer of suturing is performed for reinforcement. When an inverted Tshaped incision is made, the intersecting point should be joined firmly by $Z$ or equivalent sutures. Suture and ligation should be carefully performed to obtain accurate matching of the layered planes to avoid piercing penetration of the myometrium. These procedures are the same as in those for cesarean section in breech or transverse presentation.

\section{Postoperative Management}

When there is a cervical myoma (-Fig.9), ${ }^{4}$ outflow of lochia from the cervical canal may be inhibited by the myoma. Therefore, frequent and meticulous follow-up observations for retention of lochia are necessary. Although incarcerated uterus may undergo reduction spontaneously after cesarean section, a careful follow-up is also necessary because there is concern about retention of lochia when there are no distinct changes in cervical elongation or incarceration status.

\section{Complications}

\section{Repair of the Cut Cervical Canal}

Control of Bleeding

If there is bleeding or difficulty in delivering the fetus, it is important to secure the field of view. If the skin incision is 
narrow, and the field of view is insufficient, the incision should be extended. When the Pfannenstiel transverse incision is performed, the rectus abdominis muscle may be cut unilaterally or bilaterally (Maylard method), or the rectus abdominis fascia can be cut in a T shape toward the pubis or separated and cut in an inverted T shape. Alternatively, both of these procedures may be performed to secure the field of view.

After delivery of the fetus, it is difficult to move the uterus outside the body because of adhesions to the Douglas pouch, which makes suturing difficult. The bleeding point should be clamped with serrated forceps, and the positional relationships between the cesarean section wound, vagina, cervix, body of the uterus, and surrounding organs including the bladder, ureter, uterine artery and vein, and the cardinal ligament need to be ascertained and well understood. Bleeding from the paravaginal tissue and cardinal ligament is an important issue. If the bladder is located low or separated sufficiently, injury to the bladder and ureter is avoidable.

\section{Suture and Hemostasis of the Cervical Canal}

If the lumen of the cervical canal is obscure, orientation can be obtained by insertion of the fingers or forceps from the vagina or by insertion of a urethral balloon catheter into the cervical canal. Even when the cervical canal is cut cross-sectionally, a catheter should be passed through it, and the upper and lower cut ends can then be sutured by simple interrupted suture with 2-0 or 1-0 absorbable thread. Attention should be paid to possible rupturing of the suture after repair, and it should thus be ensured that a drain is inserted in the vicinity of the sutured portion.

\section{Injuries of the Bladder and the Ureter}

If abdominal closure is performed without recognizing the presence of injury to the bladder or ureter, an echo free space will appear in the peritoneal cavity alongside an increase in blood urea nitrogen and creatinine within a fewdays. Patients may complain of mild symptoms such as a vague feeling of discomfort and lassitude, or may sometimes be minimally symptomatic. There may also be leukocytosis and a slight increase in C-reactive protein. Caution should be exercised regarding massive transfusion-related hyperpotassemia, hepatic dysfunction, hemolytic reaction, etc.

\section{Tips and Warnings Precautions to Avoid Complications of the Bladder Injury}

When performing laparotomy for cesarean section, it is important to routinely confirm the location of the bladder.
The trainer should make sure that the trainee understands the location of the bladder, initially by direct palpation of the organ. Even when the bladder is elevated and thereby mimics the appearance of the uterine wall, manually holding it up from the both sides allows palpation and confirmation of the bladder, preventing injury to this organ.

In cesarean section cases, it is important to routinely estimate the amount of amniotic fluid, thickness of the myometrium in the lower uterine segment, space between the lowest part of the fetus and the myometrium, etc., by palpating the lowest part of the fetus via the uterine wall prior to making an incision in the myometrium. This will provide a clue as to whether, in this condition, an incision made to the depth of the estimated thickness of the myometrium would fail to reach the uterine cavity.

Conflict of Interest

None.

\section{References}

1 Takeda S. Precautions to be taken during emergency cesarean sections for breech presentation. In: Takeda S, Makino S, Takeda J, eds. Management of Breech Delivery and Shoulder Dystocia. In Japanese, Tokyo: Medical View; 2019:106-110

2 Takeda S. Education and training approaches for reducing maternal deaths in Japan. Hypertens Res Pregnancy 2018;6:15-19

3 Ishikawa G. Cesarean section for breech, transverse presentation and incarcerated uterus. In: Takeda S, Hiramatsu Y, Konishi I, Sakuragi N, eds. OGS NOW, No.3. Cesarean Section. Mastering the Essential and Practical Surgical Procedures (Japanese). Tokyo: Medical View; 2010:72-81

4 Takeda S. Cesarean section for incarcerated uterus and elongation of the uterine cervix. In: Takeda S, Hiramatsu Y, Konishi I, Sakuragi N, eds. OGS NOW, No.10. Massive Obstetric Hemorrhage: Critical Care for Intractable Bleeding and Definite Strategies of Hemostasis. In Japanese, Tokyo: Medical View; 2012:154-159

5 Sumigama S, Sugiyama C, Kotani T, et al. Uterine sutures at prior caesarean section and placenta accreta in subsequent pregnancy: a case-control study. BJOG 2014;121(07):866-874, discussion 875

6 van der Tuuk K, Krenning RA, Krenning G, Monincx WM. Recurrent incarceration of the retroverted gravid uterus at term - two times transvaginal caesarean section: a case report. J Med Case Reports 2009;3:103

7 Callen PW. Incarcerated uterus. OB-GYN Ultrasound Online 2012. http://www.fetalsono.com/teachfiles/IncarcUT.lasso

8 Gardner CS, Jaffe TA, Hertzberg BS, Javan R, Ho LM. The incarcerated uterus: a review of MRI and ultrasound imaging appearances. AJR Am J Roentgenol 2013;201(01):223-229 